\title{
Curcumin-mediated anti-microbial photodynamic therapy against Candida dubliniensis biofilms
}

\author{
Paula Volpato Sanitá ${ }^{1}$ - Ana Cláudia Pavarina ${ }^{1}$ Lívia Nordi Dovigo $^{2}$. \\ Ana Paula Dias Ribeiro ${ }^{3}$ Mariana Carvalho Andrade ${ }^{1}$. \\ Ewerton Garcia de Oliveira Mima ${ }^{1}$
}

Received: 22 September 2017 / Accepted: 5 November 2017 /Published online: 13 November 2017

(C) Springer-Verlag London Ltd., part of Springer Nature 2017

\begin{abstract}
The purpose of this study was to evaluate the effectiveness of anti-microbial photodynamic therapy (aPDT) mediated by curcumin (Cur) associated with LED light against biofilms of Candida dubliniensis, and further, investigate cellular uptake and drug penetration through the biofilms under confocal laser scanning microscopy (CLSM). Four C. dubliniensis strains were tested: three clinical isolates from HIV-positive patients and one reference strain (CBS 7987). Biofilms were treated with three Cur concentrations (20.0, 30.0 , and $40.0 \mu \mathrm{M}$ ). All samples were incubated in the dark for $20 \mathrm{~min}$ and exposed to a $5.28 \mathrm{~J} / \mathrm{cm}^{2}$ of LED light fluence. Additional samples of each strain were treated either with Cur or LED light only. Control samples had neither Cur nor light. After aPDT, results were read using the XTT salt reduction method. The data were statistically analyzed by two-way ANOVA followed by Games-Howell post-hoc test $(\alpha=$ 0.05 ). Confocal laser scanning microscopy was used to verify both the uptake of Cur by yeast cells and its penetration through the biofilm. The results showed that aPDT promoted significant reduction on the metabolism of the biofilmorganized cells of $C$. dubliniensis. Further, while Cur was rapidly taken up by $C$. dubliniensis cells, a longer time interval was required to allow Cur penetration into biofilm cells. Based
\end{abstract}

Ewerton Garcia de Oliveira Mima ewertonmima@foar.unesp.br

1 Department of Dental Materials and Prosthodontics, School of Dentistry, Araraquara, São Paulo State University (UNESP), Rua Humaitá, n 1680, Araraquara, SP 14801-903, Brazil

2 Department of Social Dentistry, School of Dentistry, Araraquara, São Paulo State University (UNESP), Rua Humaitá, 1680, Araraquara, SP 14801-903, Brazil

3 Department of Restorative Dental Sciences, College of Dentistry, University of Florida, Center Dr. 1395, Gainesville, FL 32610, USA on these results, aPDT associating LED and Cur presents promising potential on fungal control of biofilms of C. dubliniensis.

Keywords Photochemotherapy · Biofilm · Curcumin · Candida dubliniensis $\cdot$ Light-emitted diode

\section{Introduction}

Although Candida species are frequently isolated from human oral cavity as commensal microorganisms, they are important causative agents of healthcare-associated infections, especially in critically-ill immunocompromised patients [1]. In terms of epidemiology, Candida albicans remains the predominant cause of local and invasive candidiasis and accounts for more than $50 \%$ of all cases [2-5]. However, the literature has shown that the epidemiology of Candida infections has changed with emergence of non-albicans species, which have been increasingly described in both compromised and non-compromised hosts [2-5]. In vivo studies demonstrated that multiple isolations of Candida species, including C. albicans, Candida glabrata, Candida tropicalis, Candida krusei, and Candida parapsilosis, are common in patients with oral and systemic infections, both as single cultures and in mixed biofilms [3-8]. Another Candida spp. that requires attention is Candida dubliniensis. This species was recovered primarily from superficial oral candidiasis in HIV-infected patients in 1995 and its most important feature is the phenotypic similarities to $C$. albicans [9]. Due to this fact, $C$. dubliniensis can be misidentified in routine laboratory methods, and, thus, its incidence of infection may be underreported. According to the literature, $C$. dubliniensis has been isolated from local and systemic infections of diabetes mellitus patients [10], cancer patients [11], cystic fibrosis patients [12], 
immunocompromised patients [13], and immunodeficiency virus-infected $(\mathrm{HIV}+)$ patients [14-16]. This incidence must be highlighted because Candida infections in these patients may be more difficult to eradicate and, consequently, can become life-threatening [13].

One of the strategies used by several Candida spp., including C. dubliniensis, to cause infections in both compromised and non-compromised hosts is its ability to form biofilms [17]. Biofilms are biological communities with a high degree of organization, in which microorganisms form structured, coordinated, and functional communities surrounded by a self-derived extracellular matrix [18]. In this mode of growth, biofilms act as reservoirs of pathogenic microorganisms [6-8] favoring the dissemination of infections to other body sites [19]. The clinical relevance of the presence of a biofilm in an infection is that its cells are phenotypically distinct from their "free-living" or planktonic counterparts, exhibiting elevated resistance to host defenses and higher tolerance to anti-microbial agents [18]. C. dubliniensis as biofilm increases the therapeutic complexity of infections. It is not uncommon the failure of the traditional approaches available to the management of these infections due to the ability of this species to rapidly develop resistance to antifungal drugs commonly used in clinical practice $[13,15,16,20$, 21]. In fact, the recovery of some $C$. dubliniensis isolates with lower susceptibility to echinocandins [21] and resistant to azoles $[13,15,16,20]$ has been reported.

In order to overcome these problems, there is a strong medical motivation for the development of novel anti-fungal biofilm strategies. In this context, anti-microbial photodynamic therapy (aPDT), a treatment modality that has been studied for more than a century against tumoral cells [22], has shown to be effective against several pathogenic microorganisms [23-28]. In addition, aPDT has been considered as an alternative treatment modality for resistant strains [26, 28]. To fill its skills, the employment of a photoactive drug (named as photosensitizer-PS) and a visible light is needed [29]. When exposed to a specific wavelength in the presence of oxygen, the PS generates reactive oxygen species (ROS) that are toxic to cells, promoting cell death [29]. Unlike most anti-fungal drugs that act over a single cell target, the ROS interact with different cell structures [29].

Many authors have focused in searching for a PS that does not cause undesirable side effects to host's healthy cells and, for this purpose, natural products are one of the most searched ones. Curcumin (Cur) is a yellow-orange dye extracted from the root of Curcuma longa $\mathrm{L}$. and is commonly used as a spice in traditional Asian cookery [30]. It has already been reported that Cur has several pharmacological effects such as anti-inflammatory, anti-tumoral, anti-fungal, anti-bacterial, and anticarcinogenic properties [23-27, 30]. Some of which properties can be enhanced after light activating [24]. Also, phototoxicity of Cur has already been demonstrated against bacterial systems [27] and, also, against C. albicans [23]. As C. albicans showed to be more resistant to aPDT when compared to Gram-positive or Gram-negative bacteria [31], the knowledge of the effects of light-activated Cur against other Candida spp. may lead to better understanding of the potential of Cur-mediated aPDT. In this context, previous studies evaluated the anti-microbial effective of Curmediated aPDT against planktonic cultures and biofilms of both reference strains and clinical isolates of two non-albicans species-C. glabrata and C. tropicalis [23, 24]. When considering C. dubliniensis species, to the best of our knowledge, there is only one investigation that evaluated Cur-mediated aPDT against planktonic and biofilm cultures of a single reference strain [27]. Although promising results were found [27], it is also important to evaluate the effect of this treatment modality against clinical isolates, since the virulence of each strain vary according to the isolate itself, its species, and its source (ATCC $\times$ clinical) $[23,24]$. Therefore, the purpose of this study was to evaluate the effectiveness of Cur-mediated aPDT on photoinactivation of mature biofilms of three clinical isolates of $C$. dubliniensis obtained from HIV-infected patients. Additionally, this study aimed to evaluate the uptake and biofilm penetration of Cur under fluorescence imaging on confocal laser scanning microscopy (CLSM).

\section{Materials and methods}

\section{Clinical isolates of $C$. dubliniensis}

In the present study, three clinical isolates of $C$. dubliniensis obtained from the yeast culture collection of the Laboratório Especial de Micologia-UNIFESP, São Paulo, SP, Brazil, were used (C. dubliniensis $\mathrm{CD} 6$; $C$. dubliniensis $\mathrm{CD} 7$; $C$. dubliniensis $\mathrm{CD} 8$ ). These isolates were previously recovered from the oral cavity of HIV-positive patients. Additionally, a reference strain was used as control (C. dubliniensis CBS 7987). All isolates were maintained in yeast-peptone-glucose medium (YEPD: $1 \%$ yeast extract, $2 \%$ Bacto peptone, and $2 \%$ D-glucose, $2 \%$ agar) with glycerol and frozen at $-70{ }^{\circ} \mathrm{C}$ until use.

\section{Photosensitizer and light source}

The photosensitizer Cur (Sigma-Aldrich, Saint Louis, MO, USA) was used and prepared with $10 \%$ dimethyl sulfoxide (DMSO) to originate a stock solution.

A light-emitting diode (LED, LXHL- PR09, Luxeon1 III Emitter, Lumileds Lighting, San Jose, CA, USA) was used to activate the curcumin. The LED device emits $22.0 \mathrm{~mW} / \mathrm{cm}^{2}$ of light intensity and $455 \mathrm{~nm}$ of predominant wavelength.

\section{Planktonic cultures and aPDT}

In order to determine the concentrations that would be inhibitory to biofilms, an experiment was conducted with 
planktonic cultures of $C$. dubliniensis. Thus, from the stock solution of Cur, solutions were prepared at final concentrations of 5.0, 10.0, and $20.0 \mu \mathrm{M}$.

For the planktonic assays, a $25 \mu \mathrm{L}$ aliquot of each yeast was subcultured onto Sabouraud Dextrose Agar (SDA, Acumedia Manufactures Inc, Lansing, Michigan, USA) with chloramphenicol and incubated for $48 \mathrm{~h}$ at $37^{\circ} \mathrm{C}$. To prepare the yeasts' inoculums, colonies from the agar stock cultures recently cultivated were transferred using a sterile loop to a Falcon tube containing $10 \mathrm{~mL}$ of Tryptic Soy Broth (TSB, Acumedia Manufactures Inc, Lansing, Michigan, USA) and grown aerobically overnight at $37^{\circ} \mathrm{C}$. After the incubation period, each culture tube was centrifuged at $4000 \mathrm{rpm}$ for $7 \mathrm{~min}$, the supernatants were discarded, and the cell pellets were washed twice by centrifugation in $10 \mathrm{~mL}$ of sterile saline solution. Then, all washed suspensions were spectrophotometrically standardized at an optical density of $520 \mathrm{~nm}$ (Biospectro, Equipar Ltda, Curitiba, PR, Brasil) to a final concentration of $10^{6}$ cells $/ \mathrm{mL}$.

Aliquots of $100 \mu \mathrm{L}$ of C. dubliniensis standardized suspension of $10^{6}$ cells $/ \mathrm{mL}$ were individually transferred to separate wells of a 96-well microtiter plates. After inoculation on the microtiter plates, an equal volume of the three Cur solutions was added to each well (experimental conditions aPDT 5, aPDT 10, and aPDT 20). After dark incubation for $20 \mathrm{~min}$ (pre-irradiation time), the $C$. dubliniensis suspensions were irradiated on the LED device for $4 \mathrm{~min}$, which corresponds to $5.28 \mathrm{~J} / \mathrm{cm}^{2}$ light dose.

To determine whether LED light alone had any effect on cell viability, additional samples were prepared without the PS (experimental condition P-L+). The effect of Cur alone was also determined by exposing the yeast suspensions to the photosensitizer identically to those described above, but without light exposure (experimental conditions $\mathrm{P}+\mathrm{L}-5, \mathrm{P}+\mathrm{L}-10$, and $\mathrm{P}+\mathrm{L}-20$ ). The microtiter plates containing the no-light samples were kept in the dark for $24 \mathrm{~min}$, corresponding to the pre-incubation time plus light exposure time. The suspensions exposed to neither LED light nor Cur acted as overall control (experimental condition P-L-). Five samples were tested for each $C$. dubliniensis yeast, considering each experimental condition, in three independent experiments.

After all assays, tenfold serial dilutions were generated from the fungal suspensions $\left(10^{-1}, 10^{-2}\right.$, and $\left.10^{-3}\right)$ and plated on SDA in duplicate. The plates were then aerobically incubated at $37^{\circ} \mathrm{C}$ for $48 \mathrm{~h}$. After incubation, yeast colony counts of each plate were quantified using a digital colony counter (CP 600 Plus, Phoenix Ind Com Equipamentos Científicos Ltda, Araraquara, SP, Brazil) and colony forming unit per milliliter $(\mathrm{CFU} / \mathrm{mL})$ was determined.

\section{Biofilms and aPDT}

A loopful of the recently grown yeasts in SDA with chloramphenicol was individually transferred to a Falcon tube containing RPMI 1640 (Sigma-Aldrich, Saint Louis, MO, USA) and incubated overnight in an orbital shaker (AP 56, Phoenix Ind Com Equipamentos Científicos Ltda, Araraquara, SP, Brazil) at $120 \mathrm{rpm}$ and $37^{\circ} \mathrm{C}$. Then, cultures were centrifuged, the supernatants were discarded, and the cell pellets were washed twice by centrifugation in $10 \mathrm{~mL}$ of PBS and resuspended in PBS. Standardized cell suspensions at $10^{6}$ cells $/ \mathrm{mL}$ were obtained as described for the planktonic cultures.

Aliquots of $100 \mu \mathrm{L}$ of each C. dubliniensis standardized suspensions were transferred to a 96 -well microtiter plate and incubated for $90 \mathrm{~min}$ at $37^{\circ} \mathrm{C}$ in the orbital shaker at $75 \mathrm{rpm}$ for the adhesion phase of biofilm formation. After this period, each well was washed twice with PBS and $150 \mu \mathrm{L}$ of freshly prepared RPMI 1640 was added to each well. The plates were incubated for $48 \mathrm{~h}$ at $37{ }^{\circ} \mathrm{C}$ in order to generate single-species biofilms. After incubation, the wells were carefully washed twice with $200 \mu \mathrm{L}$ of PBS to remove non-adherent cells.

To evaluate the anti-fungal efficacy of the treatments, aliquots of $150 \mu \mathrm{L}$ of Cur at 20,30, and $40 \mu \mathrm{M}$ were added to each appropriate well directly over the biofilm (experimental conditions aPDT 20, aPDT 30, and aPDT 40). After $20 \mathrm{~min}$ in the dark, biofilms were illuminated $\left(5.28 \mathrm{~J} / \mathrm{cm}^{2}\right)$. To determine the effect of LED light and Cur alone on biofilm viability, the same experimental conditions for planktonic cultures were tested: P-L+, P + L- 20, P + L- 30, P + L- 40. A control condition without exposure to LED light or Cur was also conducted (P-L- group). Five samples were made for each C. dubliniensis yeast, considering each experimental condition, in three independent experiments.

The anti-fungal effects of Cur-mediated aPDT against the biofilms were evaluated by a metabolic assay based on the reduction of XTT (Sigma-Aldrich, St Louis, MO), a tetrazolium salt. After all experimental procedures, $158 \mu \mathrm{L}$ of PBS with $200 \mathrm{mM}$ glucose, $40 \mu \mathrm{L}$ of XTT, and $2 \mu \mathrm{L}$ of menadione were inoculated to each well. The plates were covered with aluminum foil and incubated for $3 \mathrm{~h}$ in the dark at $37{ }^{\circ} \mathrm{C}$. The resulting colorimetric changes are proportional to the number of living cells and their metabolic activity. Aliquots of $100 \mu \mathrm{L}$ of the reacted XTT salt solution were transferred to a new 96-well microtiter plate and the cell viability was analyzed by proportional colorimetric changes and light absorbance measured by a microtiter plate reader (Thermo Plate-TP Reader) at $492 \mathrm{~nm}$.

\section{Confocal laser scanning microscopy observations}

In order to evaluate the uptake of Cur by yeast cells, C. dubliniensis ATCC in planktonic cultures was prepared as described above and placed on $10-\mathrm{mm}$ round coverslips. Confocal microscopy was performed on live organisms. Following 5 and 20 min of incubation with Cur $20 \mu \mathrm{M}$, the glass coverslips containing cell suspension of $C$. dubliniensis were flipped and placed on a glass-bottom and observed using a Leica TCS SPE confocal microscope (Leica Microsystems 
GmbH, Wetzlar, Germany). Transmission mode using differential interference contrast (DIC) technique was used to image the fungal cells. Fluorescence mode used a $405-\mathrm{nm}$ excitation wavelength and a green fluorescence (emission from 450 to $600 \mathrm{~nm}$ ). Corresponding fluorescence and transmission images were overlayed to demonstrate Cur localization and uptake by the cells.

Cur penetration through the biofilm was also evaluated under microscope observations. Standardized suspensions were prepared as described before and biofilm formation was performed on polymethylmetacrylate coverslips. After $48 \mathrm{~h}$, the biofilms were washed twice with PBS and incubated with Cur $40 \mu \mathrm{M}$ for $20 \mathrm{~min}$. Stained biofilms were observed under fluorescence mode. Corresponding Cur fluorescence allows observation of stained cells of the biofilms. Serial sections in the $x y$ plane were obtained at $1 \mu \mathrm{m}$ intervals along the $z$ axis.

\section{Statistical analysis}

Data from planktonic cultures (CFU/mL values) were analyzed descriptively. The response variable from biofilm testing was the absorbance values from XTT (Abs XTT) while the factors analyzed were the different experimental conditions (P-L+, P + L- 20, P + L- 30, P + L- 40, P-L-, aPDT 20, aPDT 30, and aPDT 40) and the sources of the C. dubliniensis isolates (ATCC and three clinical isolates from HIV-infected patients). A twoway ANOVA was used to assess the possible effects of the factors "experimental conditions" and "sources of isolates" on XTT values. Significant differences were explored by Games-Howell post-hoc test. All tests were performed using the SPSS statistical software package (SPSS Inc., Chicago, USA) with a confidence level of $95 \%$.

\section{Results}

\section{Anti-fungal effect of aPDT against planktonic cultures of C. dubliniensis}

The microbial growth observed for all experimental conditions and C. dubliniensis strains are presented in Table 1 . The mean values of CFU $/ \mathrm{mL}$ of $C$. dubliniensis exposed only to irradiation (P-L+) or only to Cur $(\mathrm{P}+\mathrm{L}-5, \mathrm{P}+\mathrm{L}-$ 10 , and $\mathrm{P}+\mathrm{L}-20$ ) seemed similar to the values from the control group (P-L-). Only the association of Cur and LED light was capable to cause a reduction in CFU values from $10^{6}$ to $10^{3}$ (CD6 strain) and $10^{2}$ (CD7 and CD8 strains). When the aPDT groups were evaluated, it could be seen that $20.0 \mu \mathrm{M}$ of Cur was the only concentration that resulted in absence of colony growth of all $C$. dubliniensis isolates. Thus, the Cur concentrations selected to evaluate the effectiveness of Cur-mediated aPDT against $C$. dubliniensis biofilms were 20,30 , and $40 \mu \mathrm{M}$.
Table 1 Mean CFU $/ \mathrm{mL}$ values of the planktonic cultures of all C. dubliniensis isolates after assays considering all experimental conditions

\begin{tabular}{lllll}
\hline $\begin{array}{l}\text { Experimental } \\
\text { conditions }\end{array}$ & \multicolumn{4}{l}{ C. dubliniensis isolates } \\
\cline { 2 - 5 } & ATCC & CD6 & CD7 & CD8 \\
\hline P-L- & $1.83 \times 10^{6}$ & $8.74 \times 10^{5}$ & $1.82 \times 10^{6}$ & $2.94 \times 10^{6}$ \\
P + L- 5 & $8.25 \times 10^{5}$ & $6.88 \times 10^{5}$ & $7.10 \times 10^{5}$ & $2.47 \times 10^{6}$ \\
P + L- 10 & $7.50 \times 10^{5}$ & $4.96 \times 10^{5}$ & $5.41 \times 10^{5}$ & $2.80 \times 10^{6}$ \\
P + L- 20 & $8.44 \times 10^{5}$ & $5.12 \times 10^{5}$ & $4.40 \times 10^{5}$ & $2.83 \times 10^{6}$ \\
P-L+ & $2.94 \times 10^{6}$ & $1.82 \times 10^{6}$ & $1.64 \times 10^{6}$ & $3.10 \times 10^{6}$ \\
aPDT 5 & ND & $8.20 \times 10^{3}$ & $3.16 \times 10^{2}$ & $4.80 \times 10^{2}$ \\
aPDT 10 & $\mathrm{ND}$ & $1.80 \times 10^{3}$ & $\mathrm{ND}$ & $\mathrm{ND}$ \\
aPDT 20 & $\mathrm{ND}$ & $\mathrm{ND}$ & $\mathrm{ND}$ & $\mathrm{ND}$ \\
\hline
\end{tabular}

$N D$ growth not detected

\section{Anti-fungal effect of aPDT against $C$. dubliniensis in biofilm cultures}

Table 2 shows the summary of the two-way ANOVA for the factors analyzed. The interaction between both factors analyzed (sources of isolates and experimental conditions), as well as each factor isolated, showed a significant effect $(p<0.0001)$ on the XTT values of $C$. dubliniensis biofilms.

The XTT mean values of all $C$. dubliniensis biofilms after exposure to the different experimental conditions are presented in Fig. 1. Considering the factor "experimental conditions" (uppercase letters), for all sources of isolates evaluated (control strain and three clinical isolates), aPDT with the three Cur concentrations (aPDT 20, aPDT 30, and aPDT 40) caused a significant reduction of biofilm viability $(p<0.001)$ in comparison to the following groups: control (P-L-), LED light alone (P-L+), and Cur alone (P + L- 20, P + L- 30, and P + $\mathrm{L}-40)$. For these aPDT groups, the mean percentage reduction ranged from 57.70 to $82.05 \%$ considering reference and clinical strains (Table 3). There were no significant differences among the three aPDT groups for any source of isolates $(0.266 \leq p \leq 1)$. When the other experimental conditions were compared, except for the clinical isolate $C$. dubliniensis CD6, the exposure to Cur alone in the highest concentrations $(\mathrm{P}+\mathrm{L}-$ 30 and $\mathrm{P}+\mathrm{L}-40$ ) significantly reduced the biofilm viability $(0.001 \leq p \leq 0.01)$ in comparison to the control group. Also, with the exception of the clinical isolate $C$. dubliniensis CD6, exposure of $C$. dubliniensis biofilms to LED light alone (P$\mathrm{L}+)$ and to Cur alone in the lowest concentration $(\mathrm{P}+\mathrm{L}-20)$ showed no significant reduction in viability compared to the control group $(0.379 \leq p \leq 1)$.

The two-way ANOVA also found a significant effect of the sources of isolates on XTT values of $C$. dubliniensis biofilms. As observed in Fig. 1 (lowercase letters), the four isolates showed different behaviors under the experimental conditions 
Table 2 Summary of the twoway ANOVA for both factors analyzed

\begin{tabular}{llllllll}
\hline Source & $\mathrm{SQ}^{*}$ & $\mathrm{DF}^{\dagger}$ & $\mathrm{MS}^{*}$ & $\mathrm{~F}$ & $\mathrm{p}$ & $\eta_{\mathrm{p}}^{2}$ & Power \\
\hline Sources of isolates & 5702 & 3 & 1901 & 133.465 & $<0.0001$ & 0.472 & 1 \\
Experimental conditions & 32.949 & 7 & 4707 & 330.508 & $<0.0001$ & 0.838 & 1 \\
Source* & 1.88 & 21 & 0.09 & 6286 & $<0.0001$ & 0.228 & 1 \\
conditions & & & & & & & \\
Error & 6.38 & 448 & 0.014 & & & & \\
Total & 243.198 & 480 & & & & & \\
\hline
\end{tabular}

${ }^{\star} S Q$ sum of squares

${ }^{\dagger} D F$ degree of freedom

* MS mean square

tested. In general, the clinical isolate CD6 tended to be less susceptible to experimental conditions, since it showed significantly higher biofilm viability after exposure to $\mathrm{P}+\mathrm{L}-20, \mathrm{P}+$ L- 30, P + L- 40, aPDT 20, and aPDT $40(0.001 \leq p \leq 0.038)$ in comparison to the other clinical isolates. In addition, there were no significant differences in biofilm viabilities among the reference strain and clinical isolates CD7 and CD8 $(0.385 \leq p \leq 0.998)$. The exceptions occurred in control group $(p=0.022)$ and aPDT 40 group $(p=0.006)$, where there were significant differences between the reference strain and clinical isolate CD7. The only group where no significant differences $(0.066 \leq p \leq 0.878)$ were found among the four isolates tested was the LED light alone group (P-L+).

\section{Uptake of curcumin by $C$. dubliniensis}

CSLM was used to investigate the uptake of Cur by the ATCC C. dubliniensis cells in planktonic cultures and its penetration through the biofilm. Figures 2 and 3 show the confocal images after incubation of planktonic cultures with Cur $20 \mu \mathrm{M}$ and biofilms with Cur $40 \mu \mathrm{M}$, respectively, for 5 and $20 \mathrm{~min}$.
From Fig. 2, it can be observed that planktonic cultures of C. dubliniensis incubated with Cur exhibited bright green fluorescence after 5 and $20 \mathrm{~min}$, with similar uptake patterns in both incubation periods.

When the penetration of Cur through the $C$. dubliniensis biofilm was evaluated (Fig. 3), it was observed that, after 5 min of incubation, a light green fluorescence was seen in specific portions of the biofilm. After 20 min of contact with the biofilms, Cur was able to sensitize a greater amount of cells of the biofilm, and further emit a more intense fluorescence.

\section{Discussion}

C. dubliniensis has been reported as the most prevalent nonalbicans species isolated from oral candidiasis in HIV patients [32]. The recovery of resistant isolates of $C$. dubliniensis to conventional therapy with clinically used anti-fungals, such as fluconazole, has been reported [13, 15, 16, 20, 21]. The resistance of microorganisms to anti-fungal drugs has an important impact on evolution of the diseases because it makes the
Fig. 1 XTT mean values of all C. dubliniensis biofilms after exposure to the different experimental conditions. Error bars represent standard deviation. On the top of the columns, different uppercase letters show significant differences among the experimental conditions; on the bottom of the columns, different lowercase letters show significant differences among the source of isolates (Games-Howell post-hoc; $\alpha=0.05$ )

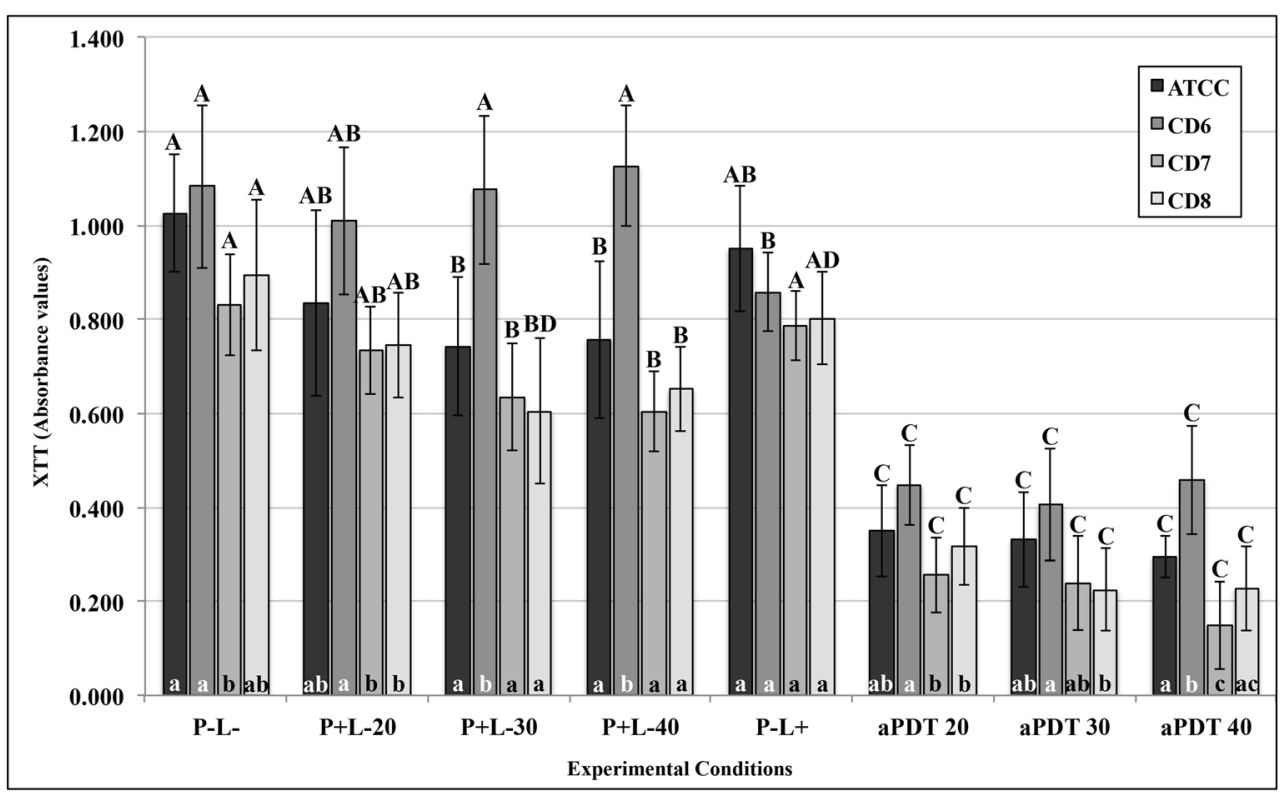


Table 3 Mean percentage reductions of all experimental groups in relation to control (P-L-)

\begin{tabular}{llllllll}
\hline Isolates & \multicolumn{2}{l}{ Mean percentage reduction } & & & \\
\cline { 2 - 7 } & P + L-20 & P + L-30 & P + L-40 & P-L+ & aPDT 20 & aPDT 30 & aPDT 40 \\
\hline ATCC & ND & 27.65 & 26.10 & ND & 65.81 & 67.56 & 71.15 \\
CD6 & ND & ND & ND & ND & 58.68 & 62.51 & 57.70 \\
CD7 & ND & 23.77 & 27.29 & ND & 69.21 & 71.19 & 82.05 \\
CD8 & ND & 32.29 & 27.11 & ND & 64.53 & 74.75 & 74.42 \\
\hline
\end{tabular}

$N D$ significant reductions were not detected in relation to P-L- $(p>0.379$ according to Games Howell post hoc test)

infections more difficult to treat, the microorganisms more challenging to eradicate, and it enhances the recurrence rates of the infections. Studies have shown that Candida isolates that have demonstrated anti-fungal resistance are susceptible to photodynamic inactivation $[26,28,33]$. Thus, this study evaluated the effectiveness of aPDT mediated by Cur associated with LED light against biofilms of three clinical isolates of $C$. dubliniensis obtained from HIV-infected patients.

In order to establish an aPDT protocol to eradicate C. dubliniensis, the planktonic cultures were submitted to different Cur concentrations and LED light, previous to the biofilm assays. The results showed that the complete inactivation of all isolates was verified only with Cur at $20 \mu \mathrm{M}$ during $20 \mathrm{~min}$ followed by $5.28 \mathrm{~J} / \mathrm{cm}^{2}$, which is in agreement with a previous study evaluating a reference strain (ATCC) of C. dubliniensis [27]. This result is also in accordance to Dovigo et al. [23], who evaluated the effectiveness of Curmediated aPDT against suspensions of $C$. albicans and observed that the same Cur concentration $(20 \mu \mathrm{M})$ was required to promote photoinactivation of the cells. Therefore, this was the lowest Cur concentration tested against biofilms in the present investigation, followed by 30 and $40 \mu \mathrm{M}$. Other studies also observed photoinactivation of suspensions of $C$. dubliniensis using Photogem® ${ }^{\circledR}[34,35]$, erythrosine [36], four dyes [37], and a natural extract of Althernathera brasiliana [38].

In the present study, mature biofilms $(48 \mathrm{~h})$ of C. dubliniensis submitted to aPDT showed significant reduction in their metabolism, regardless of the Cur concentrations (Fig. 1). When the percentage of reduction of the treatments was calculated, the clinical isolate CD6 had the lowest reductions after aPDT with the three Cur concentrations compared with the other strains, suggesting that this isolate was less susceptible to the aPDT (Table 3). In fact, the results concerning the effect of this treatment modality against the four strains evaluated (Fig. 1 and Table 3) show that there was an intra-species difference in the behavior of the isolates against the treatment. This difference may be influenced by the virulence of each strain, which vary according to the isolate itself, as well as to its source (ATCC $\times$ clinical). A previous study that evaluated a physical method of Candida spp. eradication (microwave irradiation) also detected that, after the treatment, the clinical isolates of several Candida species, including $C$. dubliniensis, had significantly higher growth $(\mathrm{CFU} / \mathrm{mL})$ than the reference strain [39]. In addition, it has been shown that the expression of some virulence factors, such as adhesion to buccal epithelial cells (BEC) and hydrolytic enzyme production [40], was also more pronounced in clinical isolates of Candida spp. from HIV patients. Another recent study also observed that a clinical isolate of $C$. albicans showed a higher adhesion force to an acrylic surface than its reference counterpart [41]. Thus, it is possible that some clinical isolates, such as the C. dubliniensis CD6 tested here, may be more pathogenic and, consequently, more difficult to eradicate.
Fig. 2 CLSM imaging of C. dubliniensis planktonic suspensions after $5 \mathrm{~min}$ (a) and $20 \mathrm{~min}$ (b) of incubation with Cur $20 \mu \mathrm{M}$. Bars show magnification of $10.0 \mu \mathrm{m}$
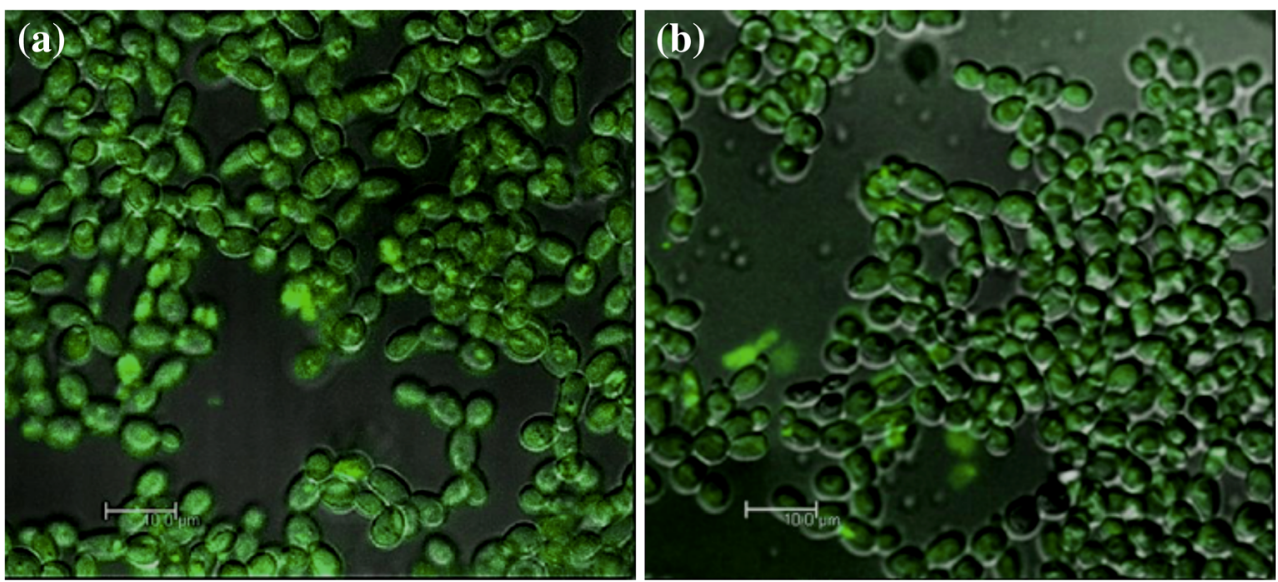
Fig. 3 CLSM imaging of C. dubliniensis biofilms after $5 \mathrm{~min}$ (a) and $20 \mathrm{~min}$ (b) of incubation with Cur $40 \mu \mathrm{M}$. In image $\mathrm{B}$, a brighter fluorescence can be seen of the biofilm than that observed in image A. Bars show magnification of $20.0 \mu \mathrm{m}$
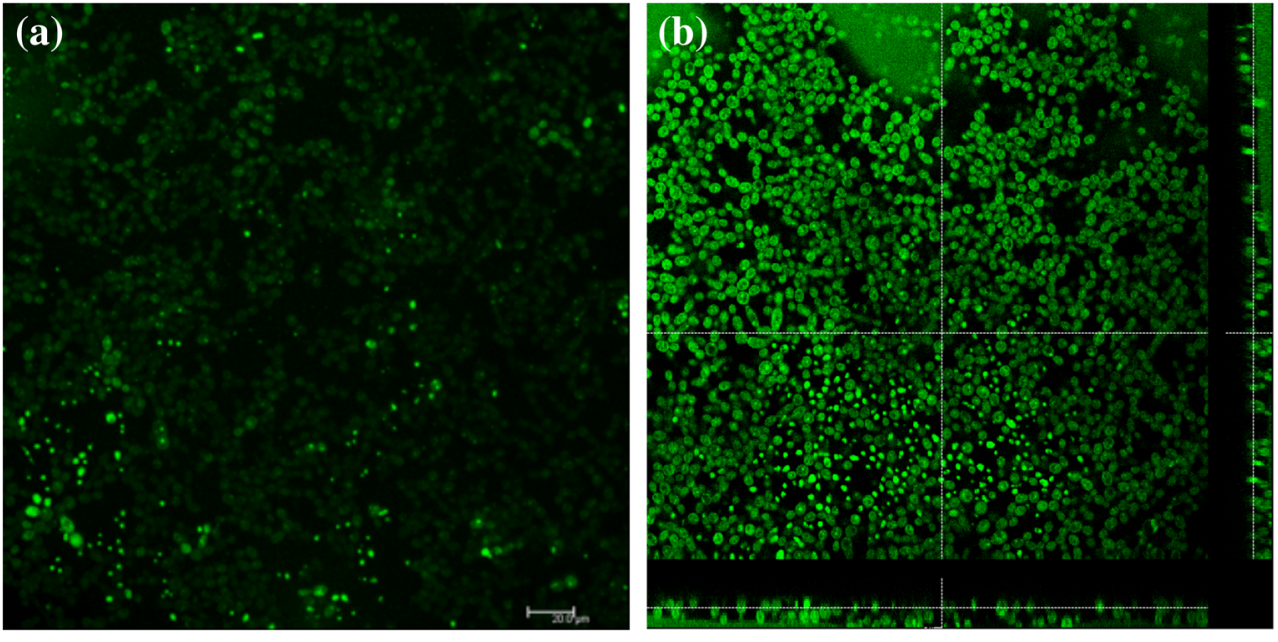

Data from the present investigation shows that, although the other C. dubliniensis strains (ATCC, CD7, and CD8) were more susceptible to aPDT with Cur at 20,30, and $40 \mu \mathrm{M}$ than CD6, the treatment with only Cur at the highest concentrations (30 and $40 \mu \mathrm{M}$ ) was also toxic to them, since small but significant reductions were observed (23.77-32.29\% / Table 3). The effect of this PS also influenced some virulence factors of Candida spp. Martins et al. [42] observed that Cur alone was able to inhibit the adhesion of Candida species to BEC, and C. dubliniensis strains isolated from the oral cavity of HIV patients showed the most significant reductions (63 and $74 \%$ ). Recently, it was also demonstrated that Cur had an anti-adhesive effect on a biofilm of $C$. albicans formed on acrylic surfaces [43]. In this study, the pre-treatment with Cur of both, disks $(800 \mu \mathrm{g} / \mathrm{mL}$ for $10 \mathrm{~min}$, corresponding to $50 \mu \mathrm{g} / \mathrm{mL}$ of Cur adsorbed to the surface) and C. albicans $(50 \mu \mathrm{g} / \mathrm{mL}$ for 3,30 , and $90 \mathrm{~min})$, significantly reduced adhesion of cells to an acrylic surface (70 and $27 \%$, respectively). Moreover, a combined pre-treatment with Cur resulted in the greatest inhibition (93\%) [43]. Those results highlight the potential of Cur as an effective anti-fungal and anti-biofilm agent.

Another important finding of the present study was the higher resistance of the biofilms to the aPDT. The results showed that Cur at $20 \mu \mathrm{M}$ for $20 \mathrm{~min}$ followed by $5.28 \mathrm{~J} /$ $\mathrm{cm}^{2}$ inhibited the growth of all $C$. dubliniensis suspensions. However, when the mature biofilms were submitted to aPDT, only a significant reduction in their metabolism was observed (Fig. 1), which is in agreement with other studies evaluating Candida spp. [23, 24, 27]. These findings may be related to the sessile organization and ecologic advantages of the biofilms, which warranty protection from the environment, nutrient availability, and metabolic cooperation [44]. In fact, biofilms are more difficult to eliminate, and drug penetration into its organized structure is equally difficult, since extracellular polymeric matrix might limit or exclude the access of drugs to the microorganisms in the deep layers of the biofilm [18].
It is important to highlight that, to aPDT promotes cell inactivation, the PS has to be bonded to or uptaken by the cells. As expected, while $C$. dubliniensis suspensions showed similar Cur uptake in both pre-irradiation times, the biofilms required a longer incubation time with Cur to be sensitized. For biofilms, the CLSM images revealed that, in contrast to the light green fluorescence emitted after 5 min of incubation with Cur (Fig. 3a), a bright green fluorescence could be seen after $20 \mathrm{~min}$ (Fig. 3b), suggesting that a longer pre-irradiation time might be required to allow penetration of Cur into the biofilm matrix and sensitization of a greater amount of cells. A similar pattern of sensitization was observed by Andrade et al. [27], who evaluated Cur uptake by biofilms of C. albicans, C. glabrata, and C. dubliniensis.

Cur is a natural pigment of low-molecular weight found in the rhizomes of turmeric and widely used as a food flavoring. Therefore, besides its therapeutic properties (anti-inflammatory, anti-microbial, anti-oxidant, and anti-cancer), its cytotoxicity may be low or improbable. Previous studies evaluated the toxicity of Cur at 5, 10, and $20 \mu \mathrm{M}$ against mammalian cells, such as macrophages [23] and fibroblasts [26], and verified that these concentrations had no toxic effect on cells metabolism. Another study evaluated the cytotoxicity of Cur at $40 \mu \mathrm{M}$ on epithelial cells (keratinocytes) co-cultured with C. albicans and verified that this concentration had no influence on the metabolism of the cells [45]. Other studies compared the susceptibility of cancer cells and mammalian cells to Cur, and they observed that breast cancer cells were 3.5-fold more susceptible than epithelial cells to Cur up to $100 \mu \mathrm{M}$ [46], and osteosarcoma cells showed higher reduction in their viability compared with osteoblast cells after incubation with Cur up to $25 \mu \mathrm{M}$ [47]. In the present study, the concentrations of Cur solution used during experiments with planktonic cells were 5,10 , and $20 \mu \mathrm{M}$, while the concentrations used in biofilms were 20,30 , and $40 \mu \mathrm{M}$. Despite these favorable results, it is important to mention that Cur at high dosages may be used with caution, since it can cause cell damage. Woo et al. verified that the treatment of 
human renal cells with Cur at $50 \mu \mathrm{M}$ for $24 \mathrm{~h}$ resulted in DNA fragmentation and apoptosis [48]. In addition, the treatment of human retinal pigment epithelial cells with Cur at concentrations among 50 and $100 \mathrm{mM}$ for $24 \mathrm{~h}$ strongly decreased the viability of the cells and increased the DNA fragmentation rate. When the higher concentration was tested $(100 \mathrm{mM})$, the treatment of the cells for 6 and $24 \mathrm{~h}$ resulted in cell apoptosis and almost no viable cells, respectively [49]. However, the susceptibility of cell culture in vitro may differ from in vivo tissue. An in vivo study demonstrated that Cur at $80 \mu \mathrm{M}$ (associated or not with light) topically applied on tongue of mice with oral candidosis showed no adverse effect on the tissue, since inflammatory response was verified in all animals with candidosis regardless the treatment received [50].

The present study showed that aPDT mediated by Cur promoted significant reductions in the metabolism of C. dubliniensis biofilms, which required a longer preirradiation time for cell photosensitization compared with the planktonic cultures. However, the viability of cells by conventional plating (CFU) was not assessed. This assessment could be important, since it has been demonstrated that biofilms submitted to aPDT may show different results when evaluated by these two viability assays (XTT and CFU) [51]. In addition, a positive control using a gold standard method of eradication of biofilm, such as chlorhexidine or an anti-fungal agent (fluconazole, nystatin), was not included in order to compare with the aPDT effect. Thus, within the limitations of this investigation, the protocol evaluated in the present study could be an alternative method for $C$. dubliniensis biofilm control and should be further tested in in vivo conditions.

Acknowledgements We would like to thank Prof. Arnaldo Lopes Colombo and Laboratório Especial de Micologia-UNIFESP, São Paulo, Brazil, for the donation of the clinical isolates of $C$. dubliniensis recovered from three HIV-positive patients, and to CEPOF RIDC FAPESP (grant number 13/07276-1) and FAPESP (grant numbers 09/17975-9 and 10/ 05425-1), for financial support. We also thank the technician's support from the laboratories.

Funding This study was funded by CEPOF RIDC FAPESP (grant number 13/07276-1) and FAPESP (grant numbers 09/17975-9 and 10/05425-1).

\section{Compliance with ethical standards}

Conflict of interest The authors declare that they have no conflict of interest.

Ethical approval The study was approved by the Ethics Committee of Araraquara Dental School (process number 16/11).

\section{References}

1. Telles DR, Karki N, Marshall MW (2017) Oral fungal infections: diagnosis and management. Dent Clin N Am 61:319-349. https:// doi.org/10.1016/j.cden.2016.12.004
2. Colombo AL, Perfect J, DiNubile M et al (2003) Global distribution and outcomes for Candida species causing invasive candidiasis: results from an international randomized double-blind study of caspofungin versus amphotericin B for the treatment of invasive candidiasis. Eur J Clin Microbiol Infect Dis 22:470-474

3. Sanitá PV, Pavarina AC, Giampaolo ET et al (2011) Candida spp. prevalence in well controlled type 2 diabetic patients with denture stomatitis. Oral Surg Oral Med Oral Pathol Oral Radiol Endod 111: 726-733. https://doi.org/10.1016/j.tripleo.2011.02.033

4. de Resende MA, de Sousa LV, de Oliveira RC et al (2006) Prevalence and antifungal susceptibility of yeasts obtained from the oral cavity of elderly individuals. Mycopathologia 162:39-44

5. Marcos-Arias C, Vicente JL, Sahand IH et al (2009) Isolation of Candida dubliniensis in denture stomatitis. Arch Oral Biol 54:127-131

6. Silva MM, Mima EG, Colombo AL et al (2012) Comparison of denture microwave disinfection and conventional antifungal therapy in the treatment of denture stomatitis: a randomized clinical study. Oral Surg Oral Med Oral Pathol Oral Radiol 114:469-479. https://doi.org/10.1016/j.00oo.2012.05.006

7. Sanita PV, Machado AL, Pavarina AC et al (2012) Microwave denture disinfection versus nystatin in treating patients with wellcontrolled type 2 diabetes and denture stomatitis: a randomized clinical trial. Int J Prosthodont 25:232-244

8. Mima EG, Vergani CE, Machado AL et al (2012) Comparison of photodynamic therapy versus conventional antifungal therapy for the treatment of denture stomatitis: a randomized clinical trial. Clin Microbiol Infect 18:E380-E388. https://doi.org/10.1111/j.14690691.2012 .03933

9. Sullivan DJ, Westerneng TJ, Haynes KA et al (1995) Candida dubliniensis sp. nov.: phenotypic and molecular characterization of a novel species associated with oral candidosis in HIV-infected individuals. Microbiology 141:1507-1521

10. Premkumar J, Ramani P, Chandrasekar T et al (2014) Detection of species diversity in oral Candida colonization and anti-fungal susceptibility among non-oral habit adult diabetic patients. J Nat Sci Biol Med 5:148-154. https://doi.org/10.4103/0976-9668.127315

11. Lai CC, Tsai HY, Chang TC, Hsueh PR (2013) Catheter-related fungemia caused by Candida dubliniensis. J Microbiol Immunol Infect 46:306-308

12. Wahab AA, Taj-Aldeen SJ, Kolecka A et al (2014) High prevalence of Candida dubliniensis in lower respiratory tract secretions from cystic fibrosis patients may be related to increased adherence properties. Int J Infect Dis 24:14-19. https://doi.org/10.1016/j.ijid.2014. 03.1380

13. Meis JFGM, Ruhnke M, De Pauw BE et al (1999) Candida dubliniensis candidemia in patients with chemotherapy-induced neutropenia and bone marrow transplantation. Emerg Infect Dis 5:150-153

14. dos Santos Abrantes PM, McArthur CP, Africa CW (2014) Multidrug resistant oral Candida species isolated from HIV-positive patients in South Africa and Cameroon. Diagn Microbiol Infect Dis 79:222-227. https://doi.org/10.1016/j.diagmicrobio.2013.09.016

15. Meiller TF, Jabra-Rizk MA, Baqui A et al (1999) Oral Candida dubliniensis as a clinically important species in HIV-seropositive patients in the United States. Oral Surg Oral Med Oral Pathol Oral Radiol Endod 88:573-580

16. Kirkpatrick WR, Revankar SG, Mcatee RK et al (1998) Detection of Candida dubliniensis in oropharyngeal samples from human immunodeficiency virus-infected patients in North America by primary CHROMagar candida screening and susceptibility testing of isolates. J Clin Microbiol 36:3007-3012

17. Alnuaimi AD, O'Brien-Simpson NM, Reynolds EC, McCullough MJ (2013) Clinical isolates and laboratory reference Candida species and strains have varying abilities to form biofilms. FEMS Yeast Res 13:689-699. https://doi.org/10.1111/1567-1364.12068 
18. Chandra J, Mukherjee PK, Leidich SD et al (2001) Antifungal resistance of candidal biofilms formed on denture acrylic in vitro. J Dent Res 80:903-908

19. Kuramitsu HK, He X, Lux R et al (2007) Interspecies interactions within oral microbial communities. Microbiol Mol Biol Rev 71: 653-670

20. Chunchanur SK, Nadgir SD, Halesh LH et al (2009) Detection and antifungal susceptibility testing of oral Candida dubliniensis from human immunodeficiency virus-infected patients. Indian J Pathol Microbiol 52:501-504

21. Pfaller MA, Jones RN, Castanheira M (2014) Regional data analysis of Candida non-albicans strains collected in United States medical sites over a 6-year period, 2006-2011. Mycoses 57:602-611. https://doi.org/10.1111/myc. 12206

22. Jori G, Fabris C, Soncin M et al (2006) Photodynamic therapy in the treatment of microbial infections: basic principles and perspective applications. Lasers Surg Med 38:468-481

23. Dovigo LN, Pavarina AC, Ribeiro AP et al (2011) Investigation of the photodynamic effects of curcumin against Candida albicans. Photochem Photobiol 87:895-903. https://doi.org/10.1111/j.17511097.2011.00937

24. Dovigo LN, Pavarina AC, Carmello JC et al (2011) Susceptibility of clinical isolates of Candida to photodynamic effects of curcumin. Lasers Surg Med 43:927-934. https://doi.org/10.1002/1sm.21110

25. Quishida CC, de Oliveira Mima EG, Jorge JH et al (2016) Photodynamic inactivation of a multispecies biofilm using curcumin and LED light. Lasers Med Sci 31:997-1009. https:// doi.org/10.1007/s10103-016-1942-7

26. Ribeiro AP, Pavarina AC, Dovigo LN et al (2013) Phototoxic effect of curcumin on methicillin-resistant Staphylococcus aureus and L929 fibroblasts. Lasers Med Sci 28:391-398. https://doi.org/10. 1007/s10103-012-1064-9

27. Andrade MC, Ribeiro AP, Dovigo LN et al (2013) Effect of different pre-irradiation times on curcumin-mediated photodynamic therapy against planktonic cultures and biofilms of Candida spp. Arch Oral Biol 58:200-210. https://doi.org/10.1016/j.archoralbio.2012. 10.011

28. Dovigo LN, Pavarina AC, Mima EG et al (2011) Fungicidal effect of photodynamic therapy against fluconazole-resistant Candida albicans and Candida glabrata. Mycoses 54:123-130. https://doi. org/10.1111/j.1439-0507.2009.01769.x

29. Konopka K, Goslinski T (2007) Photodynamic therapy in dentistry. J Dent Res 86:694-707

30. Priyadarsini KI (2009) Photophysics, photochemistry and photobiology of curcumin: studies from organic solutions, bio-mimetics and living cells. J Photochem Photobiol C: Photochem Reviews 10:81-95

31. Demidova TN, Hamblin MR (2005) Effect of cell-photosensitizer binding and cell density on microbial photoinactivation. Antimicrob Agents Chemother 49:2329-2335

32. Das PP, Saikia L, Nath R, Phukan SK (2016) Species distribution \& antifungal susceptibility pattern of oropharyngeal Candida isolates from human immunodeficiency virus infected individuals. Indian J Med Res 143:495-501. https://doi.org/10.4103/0971-5916.184288

33. Alves F, de Oliveira Mima EG, Passador RCP et al (2017) Virulence factors of fluconazole-susceptible and fluconazoleresistant Candida albicans after antimicrobial photodynamic therapy. Lasers Med Sci 32:815-826. https://doi.org/10.1007/s10103017-2177-y

34. Dovigo LN, Pavarina AC, Ribeiro DG et al (2010) Photodynamic inactivation of four Candida species induced by photogem(®). Braz J Microbiol 41:42-49. https://doi.org/10.1590/S151783822010000100009
35. Mima EG, Pavarina AC, Ribeiro DG et al (2011) Effectiveness of photodynamic therapy for the inactivation of Candida spp. on dentures: in vitro study. Photomed Laser Surg 29:827-833. https://doi. org/10.1089/pho.2011.3022

36. Costa AC, de Campos Rasteiro VM, Pereira CA, da Silva Hashimoto ES (2011) Susceptibility of Candida albicans and Candida dubliniensis to erythrosine- and LED-mediated photodynamic therapy. Arch Oral Biol 56:1299-1305. https://doi.org/10. 1016/j.archoralbio.2011.05.013

37. Hosseini N, Yazdanpanah S, Saki M et al (2016) Susceptibility of Candida albicans and Candida dubliniensis to photodynamic therapy using four dyes as the photosensitizer. J Dent (Shiraz) 17:354 360

38. Andreazza NL, de Lourenco CC, Siqueira CA et al (2013) Photodynamic inactivation of yeast and bacteria by extracts of Alternanthera brasiliana. Curr Drug Targets 14:1015-1022

39. Sanitá PV, Vergani CE, Giampaolo ET et al (2009) Growth of Candida species on complete dentures: effect of microwave disinfection. Mycoses 52:154-160. https://doi.org/10.1111/j.1439-0507. 2008.01558.x

40. Mane A, Gaikwad S, Bembalkar S, Risbud A et al (2012) Increased expression of virulence attributes in oral Candida albicans isolates from human immunodeficiency virus-positive individuals. J Med Microbiol 61:285-290. https://doi.org/10.1099/jmm.0.036269-0

41. Aguayo S, Marshall H, Pratten J et al (2017) Early adhesion of Candida albicans onto dental acrylic surfaces. J Dent Res 96: 917-923. https://doi.org/10.1177/0022034517706354

42. Martins CVB, Silva DL, Neres ATM et al (2009) Curcumin as a promising antifungal of clinical interest. J Antimicrob Chemother 63:337-339. https://doi.org/10.1093/jac/dkn488

43. Alalwan H, Rajendran R, Lappin DF et al (2017) The anti-adhesive effect of curcumin on Candida albicans biofilms on denture materials. Front Microbiol 8:659. https://doi.org/10.3389/fmicb.2017. 00659

44. Jabra-Rizk MA, Falkler WA, Meiller TF (2004) Fungal biofilms and drug resistance. Emerg Infect Dis 10:14-19

45. Pellissari CV, Pavarina AC, Bagnato VS et al (2016) Cytotoxicity of antimicrobial photodynamic inactivation on epithelial cells when co-cultured with Candida albicans. Photochem Photobiol Sci 15: 682-690. https://doi.org/10.1039/c5pp00387c

46. Ramachandran C, You W (1999) Differential sensitivity of human mammary epithelial and breast carcinoma cell lines to curcumin. Breast Cancer Res Treat 54:269-278

47. Chang R, Sun L, Webster TJ (2014) Short communication: selective cytotoxicity of curcumin on osteosarcoma cells compared to healthy osteoblasts. Int J Nanomedicine 9:461-465. https://doi.org/ 10.2147/IJN.S55505

48. Woo JH, Kim YH, Choi YJ et al (2003) Molecular mechanisms of curcumin-induced cytotoxicity: induction of apoptosis through generation of reactive oxygen species, down-regulation of $\mathrm{Bcl}-\mathrm{XL}$ and IAP, the release of cytochrome $\mathrm{c}$ and inhibition of Akt. Carcinogenesis 24:1199-1208

49. Hollborn M, Chen R, Wiedemann P et al (2013) Cytotoxic effects of curcumin in human retinal pigment epithelial cells. PLoS One 8: e59603. https://doi.org/10.1371/journal.pone.0059603

50. Dovigo LN, Carmello JC, de Souza Costa CA et al (2013) Curcumin-mediated photodynamic inactivation of Candida albicans in a murine model of oral candidiasis. Med Mycol 51: 243-251. https://doi.org/10.3109/13693786.2012.714081

51. Quishida CC, Mima EG, Dovigo LN et al (2015) Photodynamic inactivation of a multispecies biofilm using Photodithazine(®) and LED light after one and three successive applications. Lasers Med Sci 30:2303-2312. https://doi.org/10.1007/s10103-015-1811-9 